\title{
Análise Descritiva das Características Demográficas e Clínicas de Pacientes com Artrite Reumatóide no Estado de São Paulo, Brasil
}

\author{
Descriptive Analysis of the Demographical and \\ Clinical Characteristics of the Patients with Rheumatoid Arthritis \\ in the State of São Paulo, Brazil
}

\author{
Paulo Louzada-Junior ${ }^{(1)}$, Branca Dias Batista Souza $^{(2)}$, Roberto Acayaba Toledo $^{(3)}$, Rozana Mesquita Ciconelli $^{(4)}$
}

\section{RESUMO}

Objetivo: realizar uma análise retrospectiva das características demográficas e clínicas de pacientes com artrite reumatóide (AR), em acompanhamento ambulatorial no Estado de São Paulo, Brasil. Métodos: foram revisados 1.381 prontuários de pacientes com artrite reumatóide, atendidos entre 2002 e 2005 . Os prontuários foram transcritos em protocolos padronizados, coletando-se idade, sexo, raça, peso corpóreo, tempo de doença, gravidade da doença, classificação do estado funcional, fator reumatóide, condição social, presença de dor na última consulta, avaliação de progressão radiológica, envolvimento extra-articular, emprego de questionários de Qualidade de Vida ou de Atividade da Doença, tipos de tratamentos e de programa de reabilitação. Resultados: dos 1.381 pacientes, $86 \%$ eram mulheres, caucasianas, estavam entre a quarta e quinta década, tempo médio de doença de 7,2 anos e o peso corpóreo médio de $65,6 \mathrm{~kg}$. Uma minoria (5\%) foi classificada como grave, e a maioria apresentava classe funcional I e II. As manifestações extra-articulares ocorreram em $23,3 \%$, e o fator reumatóide era positivo em $71 \%$ dos pacientes. Somente um terço possuía acompanhamento radiológico e estava trabalhando. O medicamento mais utilizado foi o metotrexato (dose $15-19 \mathrm{mg} / \mathrm{semana}$ ). Um quarto dos pacientes estava freqüentando programas de reabilitação, e um terço possuía avaliações de qualidade de vida e de atividade da doença. Conclusão: essa análise proporcionou uma visão parcial dos pacientes brasileiros com $\mathrm{AR}$, identificando pontos importantes em relação às características demográficas e clínicas, os tipos de tratamentos farmacológicos e a pequena utilização de marcadores de doença e questionários de qualidade de vida, bem como a limitação de serviços de reabilitação disponíveis para os pacientes.

Palavras-chave: artrite reumatóide, São Paulo, pacientes.

\begin{abstract}
Objective: to perform a retrospective analysis of clinical and demographic characteristics of rheumatoid arthritis $(R A)$ patients followed in outpatient clinics in the State of São Paulo, Brazil. Methods: 1.381 medical records of rheumatoid arthritis patients were reviewed in the period between 2002 and 2005. These data were analyzed using a standardized form, based on the following parameters: sex, age, race, weight, follow-up time, disease progression, functional status, rheumatoid factor positivity, social status, pain in the last visit, radiologic progression, extra-articular manifestations, quantitative assessment of functional status and disease active score (DAS), pharmacological treatment, and physical therapy. Results: $86 \%$ were female Caucasians. Age of disease onset varied between the forth and fifth decades. Mean follow-up was 7.2 years and mean body weight was $65.6 \mathrm{~kg}$. Less than $5 \%$ of the patients were classified as severe and the majority of the patients presented functional class I and II. Extra-articular manifestations were observed in $23.3 \%$ and the rheumatoid factor was positive in $71 \%$. Only 33\% of the patients had radiological evaluation and were working regularly. Methotrexate was the most common medication (15-19 mg/week). Only 25\% of these patients were attending physical therapy and $30 \%$ had quantitative assessment of functional status and DAS. Conclusion: this analysis provided a partial analysis of the RA Brazilian population, identified demographic and clinical characteristics, the therapeutic drugs used, and the difficulty of the patients in attending rehabilitation services.
\end{abstract}

Keywords: rheumatoid arthritis, São Paulo, patients.

\section{Recebido em 18/01/07. Aprovado, após revisão, em 18/03/07.}

Trabalho realizado nos serviços de Reumatologia da Faculdade de Medicina de Ribeirão Preto, USP, da Faculdade de Medicina da Santa Casa de Misericórdia de São Paulo, da Faculdade de Medicina de São José do Rio Preto, da Faculdade de Medicina da Universidade Federal de São Paulo, com a coleta de dados e tabulação realizada pela Vigiun Pesquisa Clínica e Epidemiológica.

1. Professor doutor da Disciplina de Reumatologia da Faculdade de Medicina de Ribeirão Preto, Universidade de São Paulo.

2. Professora doutora da Disciplina de Reumatologia da Faculdade de Medicina da Santa Casa de Misericórdia de São Paulo.

3. Professor doutor da Disciplina de Reumatologia da Faculdade de Medicina de São José do Rio Preto.

4. Professora doutora da Disciplina de Reumatologia da Faculdade de Medicina da Universidade Federal de São Paulo.

Endereço para correspondência: Paulo Louzada Jr., Hospital das Clínicas, Disciplina de Reumatologia da Faculdade de Medicina de Ribeirão Preto, USP, Monte Alegre, Campus Universitário, Ribeirão Preto, SP, Brasil, CEP 14048-900, fax: (16) 633-6695, e-mail: plouzada@fmrp.usp.br 


\section{INTRODUÇÃO}

A artrite reumatóide (AR) é uma doença auto-imune, de caráter inflamatório e de etiologia desconhecida, caracterizada por poliartrite periférica, simétrica, que leva à deformidade e à destruição das articulações, em virtude da erosão óssea e da cartilagem. Podem ocorrer manifestações sistêmicas associadas. A maioria dos pacientes apresenta um curso clínico flutuante, com períodos de melhora e exacerbação. Com a progressão da doença, os pacientes, freqüentemente afetados em seus anos mais produtivos, desenvolvem incapacidade para realizar suas atividades, tanto da vida diária como profissional, com impacto significativo para o paciente e para a sociedade ${ }^{(1,2)}$.

Estudos epidemiológicos estimam a prevalência de AR em $1 \%$ da população adulta, afetando três vezes mais mulheres do que homens e com maior incidência entre os 35-65 anos $^{(1,3,4)}$. No Brasil, estudo multicêntrico verificou prevalência de AR do adulto variando de $0,2 \%$ a $1 \%{ }^{(5)}$.

O diagnóstico depende da associação de uma série de sintomas e sinais clínicos, achados laboratoriais e radiográficos, baseados nos critérios de classificação do Colégio Americano de Reumatologia (ACR-1987)(6). Quanto à gravidade da doença, a AR costuma ser dividida em: leve, moderada e grave. O diagnóstico precoce e o início imediato do tratamento são fundamentais para o controle da atividade da doença e para prevenir incapacidade funcional e lesão articular irreversível. Cerca de $70 \%$ dos pacientes com doença ativa desenvolvem alterações articulares dentro dos dois primeiros anos da doença ${ }^{(6)}$.

O acompanhamento e a avaliação clínica da AR, diferentemente de outras doenças crônicas como a hipertensão arterial ou hipercolesterolemia, não estão baseados apenas em uma única medida quantitativa e patognomônica. A avaliação da dor e do edema articular, da perda funcional, bem como a quantificação da proteína $\mathrm{C}$ reativa, do fator reumatóide e da velocidade de hemossedimentação, são úteis para o estabelecimento do diagnóstico, da atividade da doença e da eficácia terapêutica ${ }^{(7)}$. A avaliação do prognóstico, que detectaria tanto atividade quanto presença de lesão tecidual na $\mathrm{AR}$, inclui questionários de escore de capacidade funcional ${ }^{(8)}$.

A terapêutica do paciente varia de acordo com o estágio da doença, sua atividade e gravidade, devendo-se ser mais agressiva no tratamento quanto mais agressiva for a doen$\mathrm{ça}^{(1,2)}$. Obviamente, o tratamento, assim como a atividade da doença, devem ser constantemente reavaliados. Os principais objetivos do tratamento incluem: prevenção e controle da lesão articular, prevenção da perda de função, diminuição da dor e melhora da qualidade de vida do paciente.

Do ponto de vista de saúde pública, com o surgimento de novas abordagens terapêuticas e a progressiva preocupação com as doenças crônicas de grande impacto, o Ministério da Saúde publicou o Protocolo Clínico e Diretrizes Terapêuticas para o Tratamento da Artrite Reumatóide (Portaria MS n ${ }^{\circ} 855$ de 12 de novembro de 2002). Na mesma época, a Sociedade Brasileira de Reumatologia desenvolveu e publicou o Consenso Brasileiro para o Diagnóstico e Tratamento da Artrite Reumatóide ${ }^{(1)}$.

O desenvolvimento de novos medicamentos para o tratamento da AR, como os agentes biológicos bloqueadores do fator de necrose tumoral (TNF- $\alpha$ ), inicialmente indicados para o tratamento de pacientes com AR e persistência de doença ativa refratária ao uso das drogas modificadoras do curso da doença, reforça a necessidade da identificação de pacientes com resposta inadequada aos tratamentos habituais e/ou com progressão rápida da doença.

Apesar dos esforços para ampliar o entendimento e racionalizar o manejo da $\mathrm{AR}$, os estudos de prevalência não revelam a real situação dessas doenças em uma comunidade, pois os pacientes com doença crônica estável podem estar sendo superestimados. Uma das formas para avaliar as características dos portadores de doenças crônicas, com baixa prevalência, é a revisão de casos, retrospectiva ou prospectiva, acompanhados em serviços ambulatoriais.

Diante do exposto, o presente estudo propôs-se a coletar informações sobre as características demográficas e clínicas de pacientes com artrite reumatóide em acompanhamento ambulatorial em quatro serviços universitários de referência (dois na capital e dois no interior do Estado de São Paulo), para avaliação mais apurada de sua evolução e do estágio de tratamento.

\section{METODOLOGIA}

\section{PACIENTES}

Foram realizadas revisões de 1.381 prontuários disponíveis de pacientes com diagnóstico de artrite reumatóide, segundo os critérios de classificação diagnóstica do Colégio Americano de Reumatologia ${ }^{(4)}$, em acompanhamento nos seguintes serviços universitários: Hospital de Base da Faculdade de Medicina de São José do Rio Preto, Hospital das Clínicas da Faculdade de Medicina de Ribeirão Preto - USP, Hospital São Paulo da Faculdade de Medicina da Universidade Federal de São Paulo e Santa Casa de Misericórdia 
da Faculdade de Medicina da Santa Casa de São Paulo. Os pacientes escolhidos, identificados a partir da listagem de atendimento, tinham comparecido a uma ou mais consultas ambulatoriais entre novembro de 2002 e janeiro de 2005 . Antes de se iniciar a revisão sistemática dos prontuários, $\mathrm{o}$ estudo foi submetido e aprovado pelos Comitês de Ética em Pesquisa dos respectivos centros.

\section{INSTRUMENTOS DE COLETA}

Todos os prontuários foram transcritos em protocolos previamente padronizados. Nesses protocolos, foram anotados idade, sexo, raça, peso corpóreo, tempo de doença, gravidade da doença, classificação do estado funcional, presença do fator reumatóide, condição social do paciente, presença de dor no momento da última consulta registrada no prontuário, avaliação de progressão radiológica nos últimos 24 meses, presença de envolvimento extra-articular, emprego de questionários de Qualidade de Vida ou Escores de Atividade da Doença no seguimento, tipos de tratamentos medicamentosos utilizados e se os pacientes freqüentavam algum tipo de programa de reabilitação.

\section{RESULTADOS}

\section{DESCRIÇÃO DOS PACIENTES}

O sexo feminino representou $86 \%$ dos casos, originando relação sexo feminino/masculino de 6:1. A maioria dos pacientes era de origem caucasóide (70\%), estava entre a quarta e a quinta década de vida $(55 \%)$, compreendendo uma idade média de 53,7 anos (mediana $=54$, mínimo de 20 e máxima de 92 anos). O tempo médio de doença foi de 7,2 anos, variando de um a 51 anos. O peso médio dos pacientes observado foi de $63,9 \mathrm{~kg} \pm 13,9 \mathrm{~kg}$ (valor obtido a partir do peso de 453 pacientes) para o sexo feminino $($ mediana $=62,5 \mathrm{~kg})$ e de $74 \mathrm{~kg} \pm 16,1 \mathrm{~kg}$ para o sexo masculino (mediana $=70,8 \mathrm{~kg}$ ). Em relação à impressão clínica geral sobre o estado da doença, $45 \%$ dos pacientes foram classificados como leve e $44 \%$ como moderado, sendo que, em relação à classe funcional, a maioria foi classificada como I e II. A Tabela 1 sumariza esses resultados.

Os pacientes com idade entre a quarta e a sexta década de vida representaram a maioria nesse grupo estudado. Em relação ao tempo de diagnóstico da doença, quase a metade possuía diagnóstico de AR (46\%) há menos de cinco anos (Gráfico 1).
TABELA 1

CARACTERÍSTICAS DEMOGRÁFICAS DOS PACIENTES COM ARTRITE REUMATÓIDE $(\mathrm{N}=1.381)$

\begin{tabular}{ll}
\hline Características demográficas & Coorte de 1.381 pacientes \\
\hline Sexo feminino/masculino & $1.184(86 \%) / 193(14 \%)$ \\
Relação sexo feminino/masculino & $6: 1$ \\
Caucasóide & $930(70 \%)$ \\
Mulato & $271(20 \%)$ \\
Negro & $85(6 \%)$ \\
Asiático & $20(4 \%)$ \\
Peso ( $\mathrm{n}=453)$ & Feminino $=63,9 \pm 13,9$ \\
& Masculino $=74,0 \pm 16,1$ \\
Idade média de início da doença (anos) & $46 \pm 13,7$ \\
Tempo de doença (anos) & $7,8 \pm 7,4$ \\
Média (DP) & \\
Impressão clínica geral & Leve $=45 \%$ \\
& Moderada $=44 \%$ \\
Classe funcional & Grave $=11 \%$ \\
& I $=50 \%$ \\
& II $=37 \%$ \\
& III $=11 \%$ \\
\hline
\end{tabular}

GRÁFICO 1

DistribuiÇ̃̃o dOS PACIENTES COM AR, DE ACORDO COM O TEMPO DE DIAGNÓSTICO, DISTRIBUÍDOS EM PERÍODOS DE CINCO ANOS CADA Tempo de diagnóstico em anos $(\mathrm{n}=1.343)$

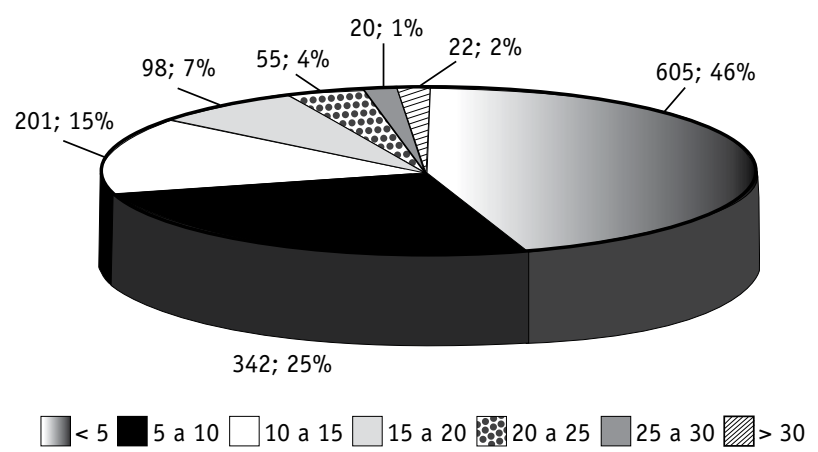

CoRRELAÇÃo ENTRE TEMPO DE DIAGNóstico E ESTADO FUNCIONAL ARTICULAR

Foi feita a correlação entre o tempo de doença e o estado funcional (Gráfico 2). Observou-se que, quanto maior o tempo de doença, maior a ocorrência de estado funcional incapacitante (III e IV).

\section{MANIFESTAÇÕES EXTRA-ARTICULARES}

Foram observadas 353 ocorrências do grupo total de pacientes definidas como manifestações extra-articulares $(23,3 \%)$. Dentre elas, a ocorrência de nódulos subcutâneos foi a mais prevalente $(29 \%)$, seguida pela presença de síndrome de Sjögren (28\%) e envolvimento pulmonar (15\%). As manifestações extra-articulares foram mais freqüentes em pacientes com quadros clínicos classificados como estado funcional articular I e II (Tabela 2). A média de positivi- 
Gráfico 2

COMPARAÇÃo ENTRE O TEMPO DE DIAGNÓSTICO DE AR (DIVIDIDO EM PERÍODOS A CADA CINCO ANOS) E O STATUS FUNCIONAL ARTICULAR $(\mathrm{N}=1.010)$

Tempo de diagnóstico $\times$ status funcional $(n=1.010)$

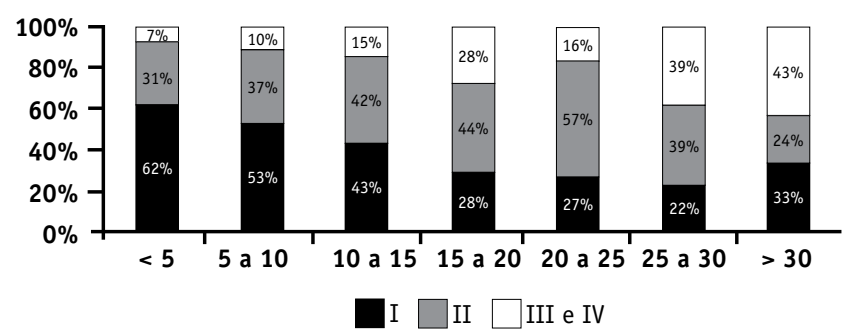

TABELA 2

PACIENTES COM AR E MANIFESTAÇÕES EXTRA-ARTICULARES $(\mathrm{N}=353)$, RELACIONADAS COM A PRESENÇA DO FATOR REUMATÓIDE E O ESTADO FUNCIONAL

\begin{tabular}{l|c|c|cccc}
\hline & & \multicolumn{6}{|c|}{ FR } & \multicolumn{5}{c}{ Status funcional } \\
Manifestação extra-articular & $\%$ & positivo \% & I \% & II \% & III \% & IV \% \\
\hline Nódulos subcutâneos & 29 & 72 & 48 & 34 & 12 & 6 \\
Síndrome de Sjögren & 28 & 74 & 49 & 38 & 11 & 2 \\
Envolvimento pulmonar & 15 & 66 & 34 & 58 & 4 & 4 \\
Neuropatia & 9 & 51 & 46 & 54 & 0 & 0 \\
Vasculite & 7 & 44 & 43 & 57 & 0 & 0 \\
Acometimento ocular & 6 & 75 & 25 & 50 & 25 & 0 \\
Síndrome de Felty & 2 & 100 & 50 & & 50 & 0 \\
Outras & 5 & & & & & \\
\hline
\end{tabular}

$\mathrm{FR}=$ fator reumatóide

dade do fator reumatóide em pacientes com manifestações extra-articulares foi de $68,2 \%$, não diferindo do observado no grupo geral, que foi de $71 \%(\mathrm{p}=0,9)$.

\section{AVALIAÇÃO RADIOGRÁFICA DO COMPROMETIMENTO ARTICULAR}

Somente um terço dos pacientes possuía acompanhamento radiológico sistemático do comprometimento articular. Ao se realizar a comparação entre o tempo de diagnóstico da doença e a evolução radiológica, observamos que, quanto maior o tempo de diagnóstico, mais grave era o comprometimento articular, avaliado pela piora radiológica, com a presença de novas erosões ósseas (Gráfico 3). Analisando de forma geral, $40 \%$ dos pacientes apresentaram piora radiológica durante o último ano analisado.

\section{ANÁLISE DA ATIVIDADE PROFISSIONAL DOS PACIENTES}

Apesar de esta análise somente utilizar informações provenientes de 650 pacientes, observou-se que somente $31 \%$ destes estavam trabalhando formalmente, e o restante ou realizavam somente atividades domésticas ou estavam afastados ou desempregados (Gráfico 4).
GRÁFICO 3

COMPARAÇÃO ENTRE O TEMPO DE DOENÇA E A PIORA RADIOLÓGICA ARTICULAR

Tempo de diagnóstico $\times$ piora radiológica $(n=314)$



$\square \operatorname{Sim} \square$ Não

GRÁFICO 4

REPRESENTAÇ̃̃O DA ATIVIDADE PROFISSIONAL DOS PACIENTES $\operatorname{COM} \operatorname{AR}(\mathrm{N}=650)$

Condição $(n=650)$

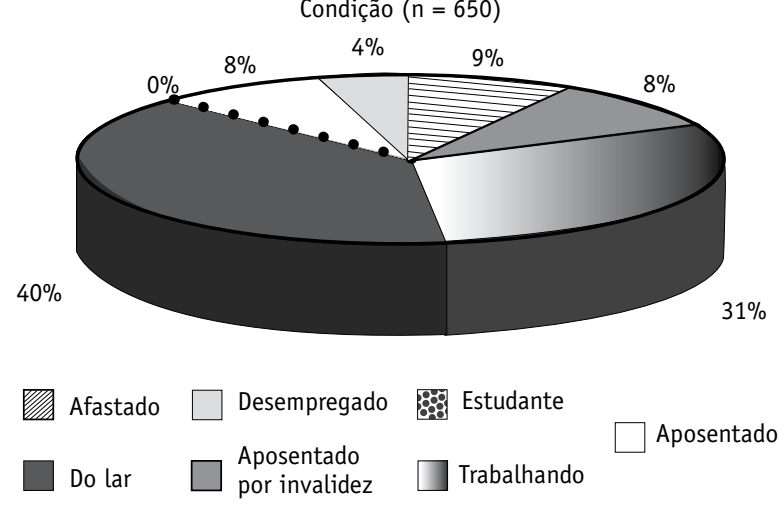

\section{ANÁLISE DA QUEIXA DE DOR}

A maioria dos pacientes (67\%) apresentava queixa de dor no momento da última consulta registrada no prontuário. Essa queixa estava presente independentemente do tempo de diagnóstico da doença. Somente $15 \%$ dos pacientes não estavam utilizando analgésicos ou antiinflamatórios.

\section{ANÁLISE DAS MEDICAÇÕES UTILIZADAS PARA O TRATAMENTO DA DOENÇA}

$\mathrm{Na}$ Tabela 3, está representado o perfil dos medicamentos utilizados para o tratamento dos pacientes com artrite reumatóide. O medicamento mais utilizado para o tratamento da AR foi o metotrexato (MTX) - 89\% dos pacientes estavam utilizando ou já o haviam utilizado. Entre os $71 \%$ dos pacientes que o estavam utilizando, a dosagem mais freqüente variava entre $15 \mathrm{mg}$ e $19 \mathrm{mg}$ por semana (38\%), seguida por entre $10 \mathrm{mg}$ e $14 \mathrm{mg}$ por semana (28\%) e entre $20 \mathrm{mg}$ e $25 \mathrm{mg}$ por semana (20\%), indicando a tendência em se utilizar doses mais elevadas do metotrexato para o tratamento da AR. Metade dos pacientes (51\%) estava utilizando somente metotrexato. Dentre as medicações combinadas ao MTX, os antimaláricos foram 
TABELA 3

PERFIL DOS MEDICAMENTOS UTILIZADOS PARA O TRATAMENTO DOS PACIENTES COM ARTRITE REUMATÓIDE $(\mathrm{N}=1.334)$

\begin{tabular}{lc}
\hline Medicamento & $\mathbf{n ~ ( \% )}$ \\
\hline Metotrexato & $500(37,4 \%)$ \\
Metotrexato e antimalárico & $315(23,6 \%)$ \\
Antimalárico & $123(9,2 \%)$ \\
AINH e/ou corticóide e/ou analgésico & $106(8,0 \%)$ \\
Leflunomida & $66(5 \%)$ \\
Metotrexato + 2 DMARD & $63(4,7 \%)$ \\
Metotrexato + leflunomida & $53(4,0 \%)$ \\
Sulfassalazina & $27(2,0 \%)$ \\
Metotrexato + sulfassalazina & $26(2,0 \%)$ \\
Leflunomida + antimalárico & $20(1,5 \%)$ \\
Metotrexato + anti-TNF & $15(1,1 \%)$ \\
Sulfassalizina + antimalárico & $11(0,8 \%)$ \\
Metotrexato + ciclosporina & $6(0,4 \%)$ \\
Ciclosporina + antimalárico & $3(0,2 \%)$ \\
\hline
\end{tabular}

AINH = antiinflamatórios não-hormonais; DMARD = drogas anti-reumáticas modificadoras da doença.

os mais observados (32\%). A leflunomida, a ciclosporina, a sulfassalazina, os agentes biológicos anti-TNF e a ciclofosfamida também foram empregados em combinação ao MTX. Alguns pacientes receberam combinação com mais de duas associações (Tabela 3 ).

Uma característica importante foi que 918 pacientes $(71 \%)$ estavam em uso de corticosteróides, por via oral, em baixas doses (dosagem variando entre $5 \mathrm{mg}$ e $7 \mathrm{mg}$ por dia).

\section{ANÁLISE DE PROGRAMAS DE REABILITAÇÃO E TRATAMENTO CIRÚRGICO}

Apenas 23\% $(\mathrm{n}=262)$ dos pacientes estavam freqüentando programas de reabilitação. Dentre esses, a maioria (92\%) freqüentava sessões de fisioterapia regularmente; $15 \%$, sessões de fisioterapia acompanhadas por terapeutas ocupacionais; e somente $1 \%$ dos pacientes fazia hidroterapia ou hidroginástica. Cerca de $11 \%$ dos pacientes com AR foram submetidos a algum tipo de tratamento cirúrgico específico para a doença articular.

\section{UTILIZAÇÃO DE QUESTIONÁRIOS DE QUALIDADE DE VIDA E DE INDICADORES DE ATIVIDADE DA DOENÇA}

Somente um terço (32\%) dos pacientes com AR possuía avaliações baseadas em questionários de qualidade de vida (HAQ). O emprego de escores de atividade da doença foi realizado em $43 \%$ dos pacientes, sendo o DAS28 e o ACR20 os mais utilizados.

\section{DISCUSSÃO}

Apesar de este estudo analisar um grupo de pacientes em seguimento em hospitais de referência e, conseqüente- mente, não representar toda a população de indivíduos com artrite reumatóide, ele se torna representativo devido ao grande número de pacientes analisados (1.381 pacientes). De interesse relevante, apesar de não ter sido mostrada a análise de cada centro individualmente, pouco diferiu o que foi observado em um único centro do observado no grupo geral. Dessa feita, consideramos que esses dados possam vir a se tornar uma referência quando quisermos traçar o perfil dos pacientes com artrite reumatóide na população do Estado de São Paulo, especialmente os pacientes atendidos em serviços terciários.

O perfil dos pacientes seria mulher (86\%), com idade variando entre 40 e 59 anos (55\%), caucasóide (71\%), pesando em torno de $65 \mathrm{~kg}$, história de início de doença há menos de dez anos (71\%), queixando-se de dor articular (67\%), em uso de analgésico ou antiinflamatório (85\%), em tratamento com metotrexato $(71 \%)$ e somente um terço trabalhando formalmente (30\%). Cerca de um quarto desses pacientes apresentava manifestações extra-articulares (23\%), sendo o fator reumatóide positivo em $70 \%$ dos pacientes. Esse perfil de paciente não difere muito do que é relatado em populações de outros países.

A observação de que, quanto menor o tempo de diagnóstico menor a prevalência de estados funcionais incapacitantes (Gráfico 2), mostra que o diagnóstico precoce, associado à terapia com drogas modificadoras da doença (DMARD), é a melhor forma de impedir a ocorrência de incapacidade física nos pacientes com AR.

As manifestações extra-articulares, excetuando as alterações laboratoriais, ocorrem em $40 \%$ dos pacientes com $\mathrm{AR}^{(9)}$. A maioria dos estudos foi realizada por meio de análise de levantamento de prontuários, com indicadores de prevalência que variavam bastante. Algumas manifestações gerais, tipo astenia, emagrecimento, anemia, foram incluídas como quadro extra-articular, enquanto, em outros estudos, somente foram computadas manifestações envolvendo órgãos ou presença de doenças associadas. A inclusão das manifestações gerais origina resultados com maior prevalência de manifestações extra-articulares. Sendo assim, como foram levantadas somente as manifestações órgão-específicas, sem considerar as gerais, observou-se que $23 \%(353 / 1370)$ dos pacientes apresentaram manifestações extra-articulares. A presença de nódulos subcutâneos foi a mais freqüente (29\%), seguida pelo envolvimento pulmonar (15\%), neuropatia periférica (9\%), vasculite cutânea (7\%) e comprometimento ocular (6\%). A síndrome de Sjögren foi a doença auto-imune reumática mais comumente associada (28\%). A ocorrência de síndrome de Sjögren secundária à ARé relatada em $50 \%$ dos pacientes ${ }^{(10)}$. 
A ocorrência de manifestações extra-articulares não diminuiu com o passar do tempo, inclusive até aumentou nessa última década, apesar dos avanços terapêuticos. Esse aumento na incidência pode ser devido a um maior número de pacientes apresentar os nódulos reumatóides por erro na identificação ou por o uso de terapia com metotrexato poder induzir a formação de nódulos. As terapias com antagonistas do TNF parecem reduzir a formação dos nódulos reumatóides. Porém, ainda há necessidade de estudos maiores para confirmar tal tendência ${ }^{(11)}$. As manifestações extra-articulares ocorrem em pacientes que apresentam uma doença articular mais grave. Porém, na nossa análise retrospectiva, os pacientes apresentavam quadro articular com predomínio das classes funcionais I e II. A freqüência de fator reumatóide positivo (70\%) não diferiu entre os grupos com ou sem manifestação extra-articular. Na verdade, são os títulos elevados de fator reumatóide que estão relacionados com a ocorrência de manifestações extra-articulares ${ }^{(12)}$. Esse dado não foi levantado na nossa análise.

A avaliação radiológica do comprometimento articular foi obtida em apenas um terço dos prontuários pesquisados. Analisando como um todo, cerca de $40 \%$ dos pacientes apresentavam piora radiológica articular, havendo uma relação direta entre o tempo de diagnóstico e o comprometimento articular, ou seja, quanto maior o tempo, pior a evolução. Essa observação somente reforça o conceito de janela de oportunidade, que enfatiza a necessidade de se iniciar o tratamento mais agressivo o mais precocemente possível ${ }^{(13)}$.

A atividade profissional estava bastante limitada nos pacientes com AR. Somente um terço estava em trabalho formal e $25 \%$ dos pacientes estavam afastados ou aposentados, mostrando o quanto a artrite reumatóide proporciona em termos de limitações à qualidade de vida pessoal e profissional, onerando a sociedade e os institutos de previdência social ${ }^{(14)}$.

A maioria dos pacientes queixava-se de dor no momento da última consulta registrada no prontuário. Essa queixa de dor não diferia em relação ao tempo de doença ou à classe funcional. $\mathrm{O}$ uso de analgésicos e antiinflamatórios também foi comumente observado (85\%), podendo ser esse um elemento agravante, em relação à ocorrência de doença péptica, com maior risco de hemorragia digestiva ${ }^{(15)}$.

Quanto à abordagem terapêutica, o estudo revela elevada frequiência de uso de analgésicos e antiinflamatórios não-hormonais (85\%), inclusive de corticosteróides (71\%). Como a ficha de coleta de dados não abrangia informações sobre a posologia dessas medicações, não é possível uma análise detalhada sobre os riscos e/ou benefícios relacionados com sua utilização na população estudada.
A revisão de prontuários confirma que o metotrexato ainda é a droga mais adotada no tratamento da artrite reumatóide nesses serviços: $89 \%$ a utilizam ou já utilizaram. Entre os $71 \%$ dos pacientes que utilizam o metotrexato, a dosagem mais freqüente variava entre $15 \mathrm{mg} \mathrm{e} 19 \mathrm{mg}$. O metotrexato é freqüentemente associado - em $49 \%$ dos pacientes - com outras drogas modificadoras do curso da doença (DMARDs). Mas, dos 978 pacientes tratados com essa droga, apenas $2 \%$ utilizam-na associada a inibidores do fator de necrose tumoral. A maioria (51\%) ainda recebe metotrexato como monoterapia. Esses achados mostram a tendência do emprego das drogas modificadoras das doenças para o tratamento da AR no Brasil, pois a maioria dos pacientes recebeu, como terapia inicial, o metotrexato em monoterapia ou combinado a outros DMARDs. O metotrexato foi a medicação inicial de escolha, com as doses consideradas moderadas/elevadas ( $86 \%$ utilizavam doses entre $10 \mathrm{mg}$ e $25 \mathrm{mg}$ por semana). Essa escolha está em concordância com as opções terapêuticas empregadas internacionalmente ${ }^{(16-18)}$.

A utilização de escores de atividade da doença e questionários de qualidade de vida $(\mathrm{QV})$ foi identificada em, respectivamente, $43 \%$ e $32 \%$ dos prontuários revisados. Se for excluído dessa análise o Serviço de Reumatologia da EPM/Unifesp, no qual 93\% dos prontuários fazem menção ao uso da ferramenta de avaliação de QV, somente $8 \%$ dos prontuários dos demais serviços mencionam o uso dessas ferramentas. Atualmente, recomenda-se a utilização dos questionários de qualidade de vida e de indicadores de atividade de doença (DAS-28 ou ACR-20, 50, 70) para acompanhar a eficácia terapêutica. Esses indicadores fornecem medidas quantitativas que podem originar instrumentos mensuráveis, tanto para uma análise objetiva do quadro da doença como para permitir que as avaliações apresentem um menor componente de subjetividade. Além disso, essas avaliações poderão ser empregadas em estudos clínicos, fornecendo parâmetros para análises posteriores. Deve-se ressaltar que vários estudos mostraram que medidas de qualidade de vida, como o HAQ, podem ser utilizadas como preditores de comprometimento funcional articular e de maior risco de morbidades associadas à artrite reumatóide ${ }^{(19-23)}$.

Com relação a tratamentos cirúrgicos e programas de reabilitação, os dados coletados revelam que $11 \%$ dos pacientes já foram submetidos a procedimentos cirúrgicos relacionados à artrite reumatóide e $23 \%$ freqüentam programas de reabilitação, principalmente fisioterapia. Esse índice é baixo, pois a maioria dos pacientes com artrite reumatóide apresenta algum tipo de incapacidade funcional durante o percurso da doençç $^{(24)}$. Sendo assim, a reabilitação torna-se imperativa, 
como uma das principais etapas da terapia. Apesar de todos os centros apresentarem serviços de reabilitação, possivelmente a grande demanda de pacientes impede que todos possam ser acompanhados, regularmente, por outros profissionais (fisioterapeutas e terapeutas ocupacionais) responsáveis pela reabilitação física destes.

Em conclusão, o presente estudo propôs-se a coletar informações sobre as características demográficas e clínicas de pacientes com artrite reumatóide, em acompanhamento ambulatorial em quatro serviços universitários de referência, para avaliação mais apurada de sua evolução e do estágio de tratamento. Apesar de ser uma análise retrospectiva, alguns pontos importantes foram identificados, especialmente em

\section{REFERÊNCIAS}

1. Laurindo IEM, Pinheiro GRC, Ximenes AC, et al: Consenso brasileiro para o diagnóstico e tratamento da artrite reumatóide. Rev Bras Reumatol 42(6): 355-61, 2002.

2. ACR Clinical Guidelins: Committee Guidelines for the management of rheumatoid arthritis. Arthritis Rheum 39(5): 713-22, 1996.

3. Symmons DPM, Barrett EM, Bankhead CR, Scott DGI, Silman AJ: The incidence of rheumatoid arthritis in the United Kingdom: results from the Norfolk arthritis register. $\mathrm{Br} \mathrm{J}$ Rheumatol 33: 735-9, 1994.

4. Ferraz MB, Ciconelli RM: Artrite Reumatóide. In: Atualização Terapêutica. São Paulo: Ed. Artes Médicas, 2003.

5. Marques Neto JF, Gonçalves ET, Barros EFO, et al: Estudo multicêntrico da prevalência da artrite reumatóide do adulto em amostras da população brasileira. Rev Bras Reumatol 33(5): 169-73, 1993

6. Arnett FC, Edworthy SM, Bloch DA, et al: The american Rheumatism Association 1987 revised criteria for the classification of rheumatoid arthritis. Arthritis Rheum 31(3): 315-24, 1988.

7. Pincus T, Sokka T: Uniform databases in early arthritis: specific measures to complement classification criteria and indices of clinical changes. Clin Exp Rheumatol 21(supl 31): 79-88, 2003.

8. Pincus T, Sokka T: Partial control of core data set measures and disease activity score (DAS) measures of inflammation does not prevent long-term joint damage: evidence from longitudinal observations over 5-20 years. Clin Exp Rheumatol 2(supl 27): 42-7, 2002.

9. Turesson C, O'Fallon WM, Crowson CS, et al: Incidence of extraarticular disease manifestations in a population based cohort of patients with rheumatoid arthritis. Arthritis Rheum (9S): S152, 2000.

10. Uhlig T, Kvein TK, Jensen JL, et al: Sicca symptoms, saliva and tear production, and disease variables in 636 patients with rheumatoid arthritis. Ann Rheum Dis 58(7): 415-22, 1999.

11. Turesson C, O'Fallon WM, Crowson CS, et al: No decrease overtime in the incidence of extraarticular disease manifestations in rheumatoid arthritis - results from a community based study of patients diagnosed during 40 years period. Arthritis Rheum 46(9): S247, 2002. relação às características demográficas e clínicas dos pacientes, os tipos de tratamentos farmacológicos utilizados e a pequena utilização de marcadores de doença e questionários de qualidade de vida, bem como a limitação de serviços de reabilitação disponíveis para os pacientes com artrite reumatóide.

\section{COORDENAÇÃO}

A coleta de dados e a tabulação dos resultados foram realizadas e coordenadas pela Vigiun Pesquisa Clínica e Epidemiológica.

Declaramos a inexistência de conflitos de interesse.

12. Turesson C, Jacobsen I, Bergstrom U, et al: Predictors of extraarticular disease manifestations in rheumatoid arthritis. Scand J Rheumatol 29(6): 358-64, 2000.

13. Quinn MM, Conaghan PG, Emery P: The therapeutic approach of early intervention for rheumatoid erthritis: what is the evidence? Rheumatology 40: 1211-20, 2001.

14. Ferraz MB, Maetzl A, Bombardier C: A summary of economic evaluations published in the field of rheumatology and related disciplines. Arthritis Rheum 40: 1587-93, 1997.

15. Laine L, Bombardier C, Hawkey CJ, et al: Stratifying the risk of NSAID-related upper gastrointestinal clinical events: results of a double-blind outcomes study in patients with rheumatoid arthritis. Gastroenterology 123: 1006-12, 2002.

16. Bresnihan B: Management of rheumatoid arthritis: synovitis. In: Hochberg MC, Silman AJ, Smolen JS, Weinblat ME, Weisman MH. Rheumatology. 3th Philadelphia: Mosby, 2003, pp. 907-13.

17. Weinblatt ME, Polisson R, Blotner SD: The effects of drug therapy on radiographic progression of rheumatoid arthritis. Results of a 36-week randomized trial comparing methotrexare and auranofin. Arthritis Rheum 36: 613-6, 1993.

18. O'Dell JR: Combination disease-modifying anti-rheumatic drug (DMASD) therapy. In: Tsokos GC (ed). Modern therapeutics in rheumatic diseases. Totoya: Human Press, 2001, pp. 147-158.

19. Wolfe F, Cathey MA: The assessment and prediction of functional disability in rheumatoid artrhritis. J Rheumatol 18: 1298-306, 1991.

20. Pincus T, Brooks RH, Callahan LF: Prediction of long-term mortality in patients with rehuamtoid arthritis according to a simple questionnaire and joint count measures. Ann Intern Med 120: 26-34, 1994.

21. Gabriel SE, Crowson CS, O'Fallon WM: Mortality in RA: have we made an impact in 4 decades? J Rheumatol 26: 2529-33, 1999.

22. Wolfe F, Michaud K, Choi HK: Predicting mortality in patients with rheumatoid arthritis. Arthritis Rheum 48: 1530-42, 2003.

23. Sokka T, Hakkinen A, Krishnan E, Hannonen P: Similar prediction of mortality by the health assessment questionnaire in patients with rheumatoid arthritis and the general population. Ann Rheum Dis 63: 494-7, 2004.

24. Eberhardt KB, Fox E: Functional impairment and disability in early rheumatoid arthritis: development of five years. J Rheumatol 22: 1037-42, 1995. 